\title{
Decision Making Capacity or How to Accept Patient's Refusal for Orthodontic Treatment
}

\author{
Yulia Bogdanova Peeva*
}

Department of Social Medicine and Public Health, Faculty of Public Health, Medical University of Plovdiv, Bulgaria

Correspondence to: Yulia Bogdanova Peeva, Department of Social Medicine and Public Health, Faculty of Public Health, Medical University of Plovdiv, Bulgaria. Received date: July 14, 2021; Accepted date: August 3, 2021; Published date: August 10, 2021

Citation: Peeva YB (2021) Decision Making Capacityor How to Accept Patient's Refusal for Orthodontic Treatment.J Med Res Surg 2(4):pp. 1-3. doi:10.52916/jmrs214054 Copyright: @2021 Peeva YB. This is an open-access article distributed under the terms of the Creative Commons Attribution License, which permits unrestricted use, distribution and reproduction in any medium, provided the original author and source are credited.

\section{ABSTRACT}

Introduction: Communication in dentistry is bilateral process which usually is based on response (understanding) by the person. That's why the Oral Healthcare Providers (OHP) should be convinced the consent given by the patient is valid. It means that at the beginning of the treatment the orthodontist will ask a lot of questions and have expectations to receive appropriate answers. There is a specific lack of awareness about the first orthodontic consultation at $7 y$ of age, occurrence and prevention of most of the common tooth jaw discrepancies which affect the oral health, self-confidence and overall development of the child. A variety of socio-demographic, educational, personal and other factors mostly divided into objective and subjective factors influences the perception of facial attractiveness. The orthodontic treatment lays down on the personal desire and attitudes, depends from the motivation but is not without a risk for the patient. The aim of the current research is to present the most objective and subjective factors identifying the patient's refusal.

Material and methods: It's a case report based on preliminary discussion and orthodontic consultation over the cephalometric analysis and cast models. Orthodontic treatment protocol was followed and given informed consent by the individual was received.

Results and discussions: An electronic search was conducted using the Medline database (PubMed), Science Direct, and Scopus. In this case report were described the treatment options for Class III malocclusion with an emphasis on maxillary protraction and existing impacted canine 13 . The decision making capacity was evaluated and also what are the objective and subjective factors and how to proceed with patient refusal.

Conclusions: Despite the orthodontist's efforts to improve the management of the dental practice and to attract new patients, these challenges should never been from the first importance. Contemporary dentistry requires that the patient's right to refuse should be respected and this refusal must be accepted. Because orthodontic treatment is expensive, the process of returning money or sharing responsibility for the treatment depend on the socio-cultural characteristics of both the patient and the doctor. The whole situation requires a very delicate approach, as it affects the image of the dental community in society at whole.

\section{Keywords:}

Competence, Patient's Motivation, Orthodontic Treatment, Patient's Refusal

\section{Introduction}

The informed consent in orthodontic treatment confirms autonomy as a basic legal and ethical principle obligatory when providing healthcare services. "Oral health is essential to general health and well-being and greatly influences quality of life. It is defined as a state of being free from mouth and facial pain, oral diseases and disorders that limit an individual's capacity in biting, chewing, smiling, speaking and psychosocial well-being". However, there is no interest for the researchers to study the feelings of an adult patient who has not undergone orthodontic treatment. Index of Orthodontic Treatment Need (IOTN) is highly valued (4-5 in a scale), ie., the objective necessity exists and is present [1]. From the literature is known, that the attractive people are seen as significantly more successful personal aspect [2]. It improves their relationships-friendship, love and collegiality-their personal success in life, their social status and quality of life. A significant aspect of side effects that the orthodontist may suggest as a "friendly colleague", unacceptable information from the Internet and the patient's personal fears about what lies ahead may negatively affect the patient and refuse treatment $[3,4]$.

\section{Case Report}

A case of a 40-years-old patient with an impacted 13 is presented [5]. Clinically shows a straight profile Class III malocclusion with anterior divergence, midface deficiency resulting in sunken appearance, relative mandibular prognathism, prominent chin, with anterior cross-bite or edge-to-edge relation, and narrow maxillary arch with an impacted canine 13 . Even though, Class III malocclusion gives senile look to young adults and they are not so attractive. The concrete patient has a specific sense of humour.

In the preliminary conversation he said: "I know, I look like Quasimodo! Iaccepted it! I do not mind!" $[6,7]$. The main question 
here is what the orthodontist will answer. Will he agrees-from a human and medical point of view it is unacceptable, because in this way it will offend him. If he denies, then the patient does not need treatment. Probably, its better not to answer at the moment. From now on, the educational stage in communication with the patient begins. The orthodontist's ability to allow the patient to see through his eyes shows enough time for awareness and more cooperative patients to less difficulties in daily work.

The initial assessment of the individual based on questions such as: "How will I work with this person in the next 2 years?", "What difficulties can he create for me?", "Is he communicative?", "Can I easily convince him?". These questions should forward the doctor to understand more about psychological, educational and other personal characteristics of the patient. Based on personal observations, the orthodontist will accept to work easier with people who have a sense of humor. In addition, elderly patients come with their own motivation, which does not depend on the persistence of the mother (With the greatest respect that every mother deserves) $[8,9]$.

A personal decision has been made, the treatment is paid in advance and the best part is ahead-the one in which the orthodontist succeeds. The tickle of satisfaction borders on vanity, and it is of the devil. It is more productive if the doctor is constantly looking for his mistakes and considering possible alternatives. The uncertainty shown to the patient is highly inadvisable. The best question in this case is: "What have to be the treatment protocol if this is my child?".

\section{Results and Discussions}

The following pictures present the need of orthodontic treatment for the patient (Figure 1-2).

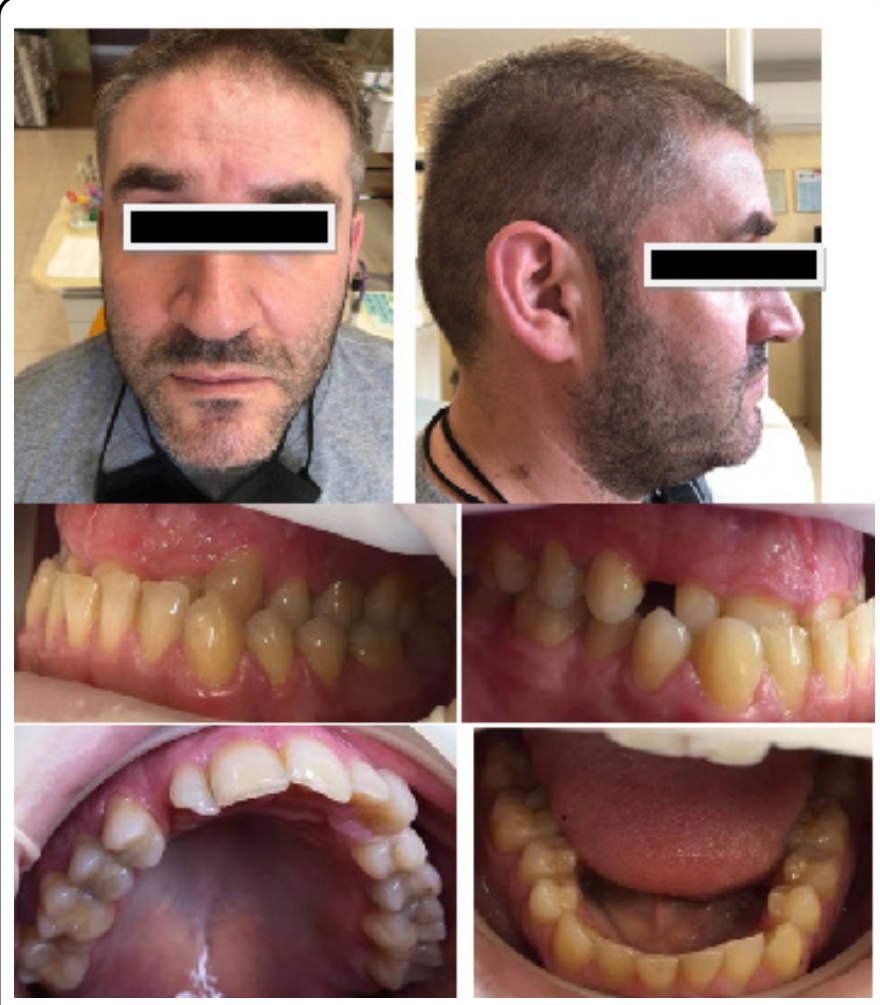

Figure 1: Pre-treatment initial face and intraoral photographs.

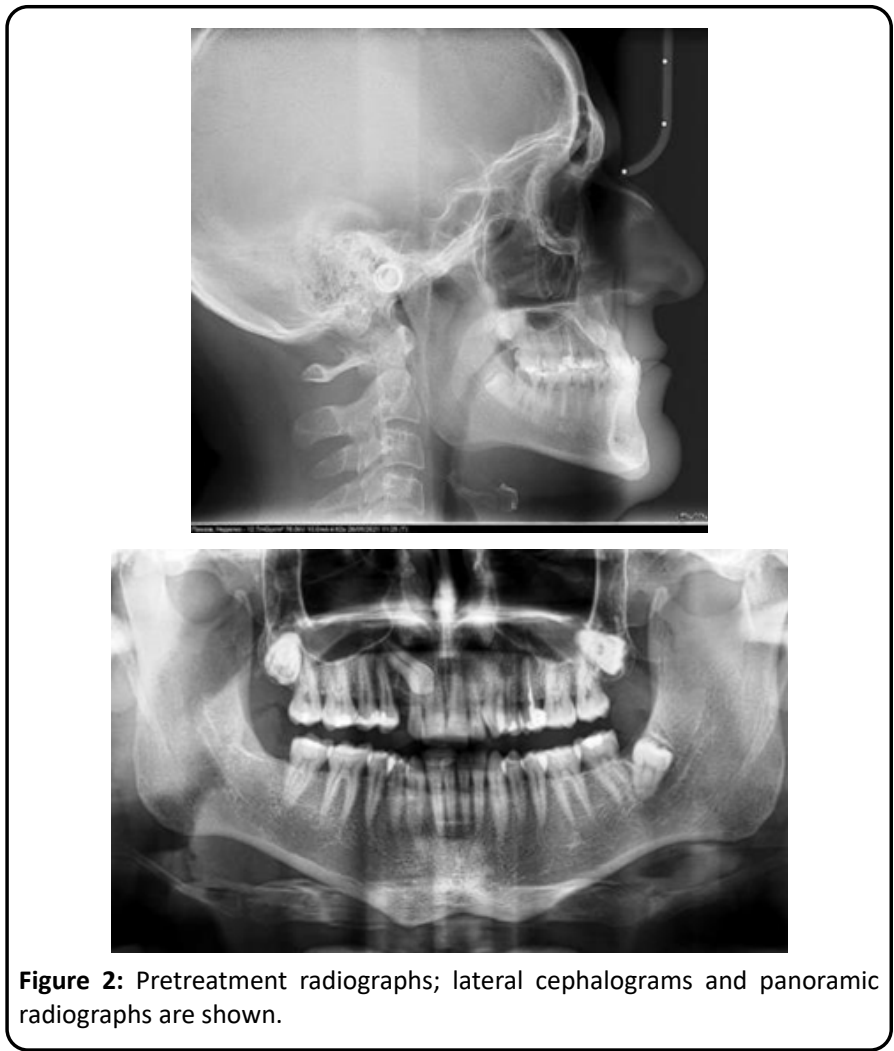

The cephalometric findings shows that this patient was diagnosed with a skeletal Class III malocclusion with mandibular protrusion, facial asymmetry, and impacted canine 13 . The following treatment protocol was planned:

- Correct the patient's facial asymmetry and coordinate the facial, maxillary, and mandibular dental midlines;

- Correct the skeletal Class III anteroposterior jaw relationship;

- Extraction of 34,44

- Extraction of $18,28,38$

- Retrieve 13

- Correct the canted occlusal plane and achieve an ideal overjet and overbite relationship [10,11] (Figure 3).

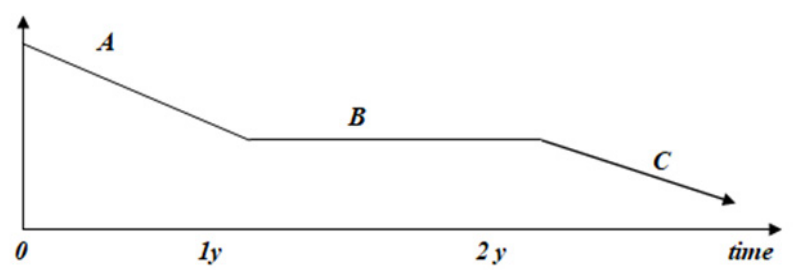

Figure 3: Acceptable motivation during 2 years of orthodontic treatment. A) Initial motivation (usually external, by parents. When the patient is child). B) Continuing motivation. C) Motivation during retainer period.

To obtain the informed consent and to keep it on the levels which still remain the initial process is an additional challenge when providing orthodontic treatment is longer then the other dental procedures. The future patients have been informed also about 'lifelong' retention management to minimize the risk of recurrence. That is the reason why cooperation and motivation is considered in so many researches [12,13] (Figure 4). 


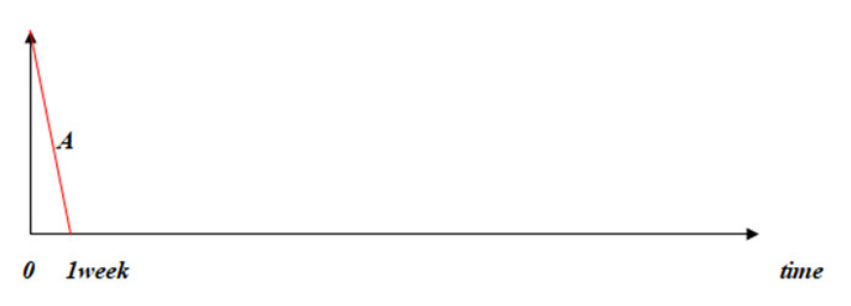

Figure 4: The initial and continuing motivation of the current patient. A) Initial motivation.

The Oral Health Impact Profile (OHIP) must also accept the idea that a valid consent is not a one-time procedural exercise "tick the box" but a continuous and educational process of providing an appropriate information to a person without medical knowledge. It means that the doctor should follow the ethical principles to keep the healthcare model.

\section{Conclusion}

- Inconvenience, subsequent worries and related issues can befall any dentist, regardless of his or her work experience and ability to communicate with patients.

- The significance of informed consent remains essential.

- The patient's ability to form his own opinion based on Internet information interferes with work.

- In his work, the dentist must defend the honor of the professional organisation, not his personal interests.

- In a conclusion, I asked him: "If you are about to have heart or abdominal surgery, would you still trust the internet?".

- These days all over the world the people show bigger respect to the doctors efforts to keep the life. And of course, that's wonderful.

\section{Conflict of Interest:}

The author do not have any conflict of interest to declare.

\section{Funding:}

None

\section{References}

1. Staudt C, Kiliaridis S (2009) Different skeletal types underlying Class III malocclusion in a random population. Am J Orthodont
Dentofac Orthop 136(5): pp. 715-721.

2. Chen $M Z$, Feng $X, L i Z$, et al. (2015) Impact of malocclusion on oral health-related quality of life in young adults. Angle Orthod 85: pp. 986-991.

3. Nanda RS, Kierl MJ (1992) Prediction of cooperation in orthodontic treatment. Am J Orthod Dentofac Orthop 102(1): pp. 15-21.

4. Livas C, Delli K, Ren Y (2013) Quality evaluation of the available Internet information regarding pain during orthodontic treatment. Angle Orthod 83: pp. 500-506.

5. De Clerck H, Nguyen T, De Paula L, et al. (2012) Threedimensional assessment of mandibular and glenoid fossa changes after bone-anchored Class III intermaxillary traction. Am J Orthodont Dentofac Orthop 142(1): pp. 25-31.

6. Almeida A, Leite I, Melgaço C, et al. (2014) Dissatisfaction with dentofacial appearance and the normative need for orthodontic treatment: determinant factors. Dent Press J Orthod 19: pp. 120-126.

7. Burden D, Pine C (1995) Self-perception of malocclusion among adoloscents. Community Dent Health 12: pp. 89-92.

8. Mandall N, Wright J, Conboy FM, et al. (2001) The relationship between normative orthodontic treatment need and measures of consumer perception. Community Dent Health 18: pp. 3-6.

9. Shaw W, Addy M, Dummer P, et al. (1986) Dental and social effects of malocclusion and effectiveness of orthodontic treatment: a strategy for investigation. Community Dent Oral Epidemiol 14: pp. 60-64.

10. Turpin D (2007) Orthodontic treatment and self-esteem. Am J Orthod Dentofac Orthop 131: pp. 571-572.

11. Brook P, Shaw W (1989) The development of an index of orthodontic treatment priority. Eur J Orthod 11: pp. 309-320.

12. Gosney M (1986) An investigation into some of the factors influencing the desire for orthodontic treatment. Br Dent J 13: pp. 87-94.

13. El Mourad AM, Al Shamrani A, Al Mohaimeed M, et al. (2021) Self-Perception of Dental Esthetics among Dental Students at King Saud University and Their Desired Treatment. Int J Dent 6671112. 